Chapter 18

\title{
Postmortem DNA: QC Considerations for Sequence and Dosage Analysis of Genes Implicated in Long QT Syndrome
}

\author{
Stella Lai, Renate Marquis-Nicholson, \\ Chuan-Ching Lan, Jennifer M. Love, Elaine Doherty, \\ Jonathan R. Skinner and Donald R. Love
}

Additional information is available at the end of the chapter

http://dx.doi.org/10.5772/51685

\section{Introduction}

Long QT syndrome is a rare disorder of cardiac ion channels, characterised by a prolonged QT interval and T-wave abnormalities on electrocardiogram (ECG) and the occurrence of the ventricular tachycardia torsade de pointes. Sodium, potassium or calcium channels present in heart muscle may be affected, altering the regulation of electrical current in the cells [1-3]. Individuals with this condition will be predisposed to cardiac events such as arrhythmias and polymorphic ventricular tachycardia, which may lead, if untreated, to sudden cardiac death [2,3]. Thirteen genes are associated with the condition, and hundreds of mutations have been identified [3-5]. Currently, more than $95 \%$ of the pathogenic mutations listed in disease databases (Gene Connection For the Heart, http://www.fsm.it/cardmoc/; online Human Gene Mutation Database, www.hgmd.cf.ac.uk/) are sequence variants (including point mutations and small insertions or deletions), but the importance of whole or multi-exon deletions and duplications has more recently been recognised [6] and it is now recommended to use both sequence and dosage techniques in order to provide comprehensive analysis [3].

In New Zealand, the majority of specimens referred for Long QT syndrome diagnostic testing are retrieved after death. Postmortem specimens are often difficult to handle as they are usually either tissue samples or severely haemolysed blood. The extracted DNA is frequently of low quality, due to the presence of unwanted material such as short fragments produced by degradation and chemical modifications from oxidation and hydrolysis processes [7]. As a result, only short sequences can be reliably amplified [7]. Moreover, capillary-based 
sequencing, the gold standard first-line diagnostic test for Long QT syndrome, is very sensitive to the presence of contaminants, such as proteins, RNA and residual salt. The presence of such contaminants leads to poor quality electropherograms for analysis, which tend to be compromised by the presence of dye blobs, C-shoulders and a variable degree of baseline noise (Figure 1). Although the QC requirements for array comparative genomic hybridisation $(\mathrm{aCGH})$, which can be used to detect whole exon deletion and duplication mutations, are less stringent than those for sequencing, contamination or degradation of sample DNA can lead to suboptimal efficiency of labelling and hybridisation. Such difficulties arising in the practical procedure mean that the analysis of postmortem DNA can be time-consuming and challenging, and obtaining high quality data within a reasonable timeframe can be extremely difficult.
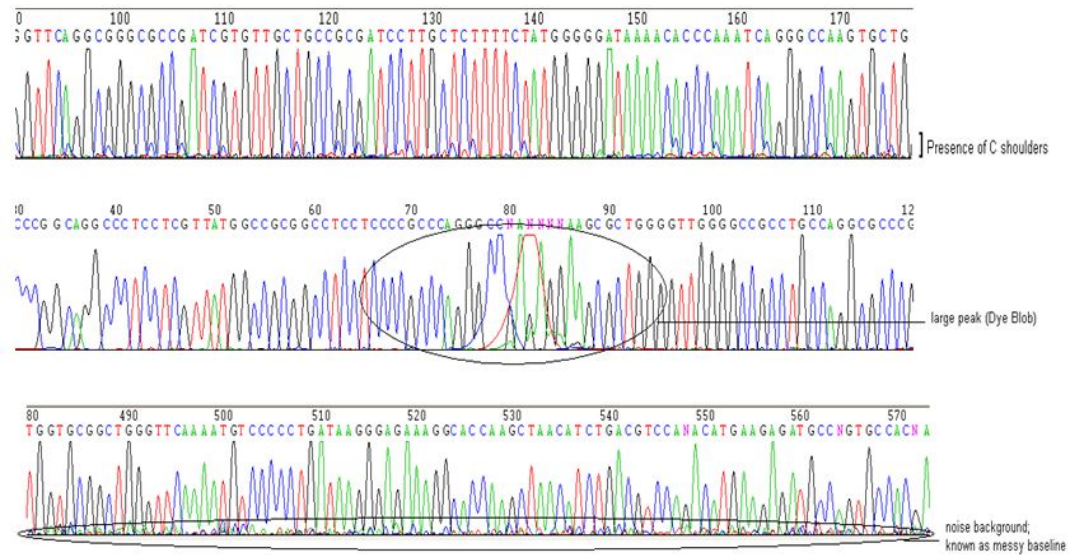

Figure 1. Electropherograms showing poor quality sequence data.

Long QT syndrome affects 1 in 2000 individuals [8] and contributes to 15-25\% of sudden unexplained death in 1-40 year olds $[9,10]$ and $10 \%$ of sudden unexplained deaths in infancy (SIDs) [11]. As a consequence, it is an important differential diagnosis to be considered in all cases of autopsy negative young sudden death. Molecular genetic testing is essential to make a postmortem diagnosis, given that screening for the electrocardiogram characteristics is no longer possible after death. Historically the turnaround time for diagnosis has been up to six months, due to the large number of genes to be analysed and the difficulties inherent in dealing with postmortem specimens (as detailed above). However, the demand by coroners for diagnostic laboratories to undertake more rapid analysis has been steadily increasing. A protocol tailored specifically to the treatment of postmortem specimens is necessary to meet this demand. Within our laboratory, we have successfully developed a robust process for sequence and dosage analysis of postmortem samples and have achieved an excellent turnaround time of 6-12 weeks. Here, we address the critical QC parameters that should be considered in order to obtain high quality data for rapid, accurate analysis. The discussion 
presented below concerns both sequence analysis and dosage analysis. The former uses conventional sequencing technology, while the latter involves the simultaneous high resolution screening of deletion and duplication mutations in multiple cardiac genes as opposed to the more conventional multiplex ligation dependent probe amplification (MLPA) technique, which many diagnostic laboratories still use [6].

\section{Materials and methods}

\subsection{Sequencing}

\subsubsection{Primer design}

We have used two approaches to design primers flanking each of the coding exons of the genes KCNQ1 (LQT1), KCNH2 (LQT2), SCN5A (LQT3), KCNE1 (LQT5), KCNE2 (LQT6), and KCNJ2 (LQT7), including at least 50 base pairs of the flanking intronic regions. The first used the primer design program called PrimerQuest (Integrated DNA Technologies Inc; http:// www.idtdna.com/Scitools/Applications/Primerquest/Advanced.aspx). This program allows the region in a sequence file to be specified, against which primers are designed to flank the targeted region. The designed primers for each exon are then checked in silico for annealing characteristics using the Macintosh-based program, Amplify. Finally, all primers were checked for single nucleotide polymorphisms (SNPs) using the software tool available from the National Genetic Reference Laboratory, Manchester (http://ngrl.man.ac.uk/SNPCheck/ SNPCheck.html). This bioinformatics program uses the current National Center for Biotechnology Information (NCBI) build of the human genome and the current release of the Single Nucleotide Polymorphism database (dbSNP) to identify the position in the sequence where the primers bind and to detect any known SNPs at these sites

Following the above approach, we developed an alternate design protocol $[12,13]$. Each mRNA sequence of interest was identified through the public UCSC genome browser page http://genome.ucsc.edu. This website provides a direct link to ExonPrimer for the design of primers specific to the mRNA sequence. ExonPrimer uses exon position information provided by the UCSC genome browser in combination with the primer design tool Primer3 to create primer pairs according to set parameters, while avoiding pairing to homologous regions within the genome. Exon and amplicon size are provided and multiple alternative primer sets are given. Following primer design, all primers were checked for single nucleotide polymorphisms (SNPs), as described above. Following a negative SNP check the primer sequences were evaluated using the UCSC genome browser to confirm the identification of single amplicons. Each primer was then tailed with an M13 sequence and manufactured by Integrated DNA Technologies Inc or Life Technologies. In this way, primers are designed to allow amplification of all exons of interest and the corresponding splice sites using a single set of PCR conditions such that PCR (and subsequent sequencing) can be performed under identical conditions within a 96-well PCR plate. 


\subsubsection{DNA extraction}

Genomic DNA (gDNA) was extracted from peripheral blood leucocytes (EDTA blood samples) using the Gentra Puregene DNA Extraction kit (Qiagen), according to the manufacturer's instructions.

A standard phenol/chloroform protease protocol was used to extract gDNA from postmortem tissue specimens. A small section $(2 \mathrm{~mm} \times 2 \mathrm{~mm} \times 4 \mathrm{~mm})$ is usually cut from frozen tissue and diced as finely as possible using a scalpel blade. The tissue is placed into a $1.5 \mathrm{ml}$ microcentrifuge tube with $450 \mu \mathrm{l}$ of $1 x$ TES Buffer (1M NaCl, $0.5 \mathrm{M}$ Tris- $\mathrm{HCl}, 10 \mathrm{mM}$ EDTA), $60 \mu \mathrm{l}$ of $20 \mu \mathrm{g} / \mu \mathrm{l}$ Proteinase K (Roche) and $10 \mu \mathrm{l}$ of $10 \%$ SDS, and incubated overnight with vigorous shaking. Following digestion, an equal volume of phenol is added and the sample is vortexed vigorously. Once homogenous, the sample is centrifuged to separate the layers and the top aqueous layer is removed and transferred to a fresh $1.5 \mathrm{ml}$ centrifuge tube. An equal volume of chloroform is then added to the aqueous layer. This is vortexed, centrifuged, and the aqueous (top) layer again transferred to a fresh tube. A $2 x$ volume of $100 \%$ ethanol is added to the aqueous layer to precipitate the DNA, followed by centrifugation at $13,000 \mathrm{rpm}$ and the supernatant is removed. The pellet is then washed with $70 \%$ ethanol and re-centrifuged for 2 minutes at 13,000rpm. The supernatant is again removed, and the pellet air dried prior to re-suspension in TE buffer (10mM Tris-HCl, 1mM EDTA, pH 7.0-8.0).

The quality and quantity of extracted gDNA is measured using a NanoDrop ND-1000 Spectrophotometer.

\subsection{3. $P C R$}

PCR amplification is performed in a final $25 \mu \mathrm{l}$ reaction volume with the following reagents: Faststart buffer (Roche Applied Science), $2 \mathrm{mM} \mathrm{MgCl}_{2}$ (Roche Applied Science), M13-tailed forward and reverse primers at $0.8 \mu \mathrm{M}$ each (synthesised by Integrated DNA Technologies Inc), $0.4 \mathrm{mM}$ dNTPs (GE Healthcare Ltd), 1 unit Faststart Taq DNA polymerase (Roche Applied Science) and $5 \mu \mathrm{l}$ GC-rich solution (Roche Applied Science). 50ng of gDNA is included in each reaction. PCR amplification is carried out with the following conditions: denaturation at $95^{\circ} \mathrm{C}$ for 5 minutes, followed by 35 cycles of $94^{\circ} \mathrm{C}$ for 45 seconds, $60^{\circ} \mathrm{C}$ for 30 seconds and $72^{\circ} \mathrm{C}$ for 30 seconds, with a final extension of $72^{\circ} \mathrm{C}$ for 10 minutes.

\subsubsection{Sequencing (Figure 2)}

$5 \mu \mathrm{L}$ of each PCR is cleaned with ExoSAP-IT (Affymetrix, USB) prior to bidirectional DNA sequencing using M13 forward and reverse primers and Big-Dye Terminator v3.0 (Applied Biosystems Ltd). 20 $\mu$ l of each sequenced product is manually purified using the CleanSEQ Sequencing Purification System (Agencourt Bioscience). Four different drying times prior to elution (20 minutes, 24 hours, four days and seven days) were assessed to establish an optimal drying time for generating high quality sequencing data. $15 \mu \mathrm{L}$ of purified product was then subjected to capillary electrophoresis using the Applied Biosystems model 3130xl Genetic Analyzer. 
The analysis of sequence traces is performed using Variant Reporter v1.0 (Applied Biosystems). Variant Reporter uses advanced algorithms and quality metrics to automate the detection of variants and to streamline the analysis process.

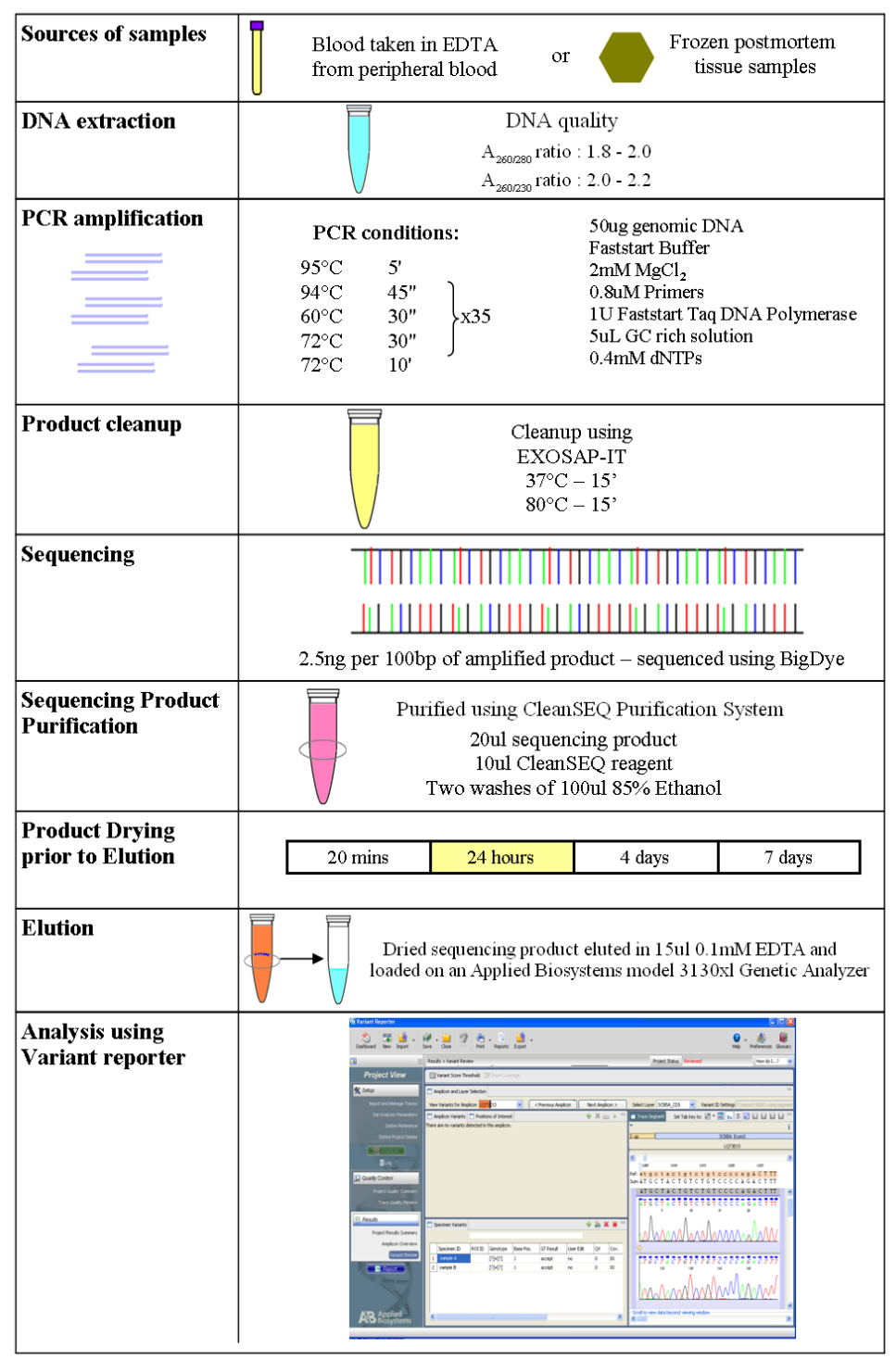

Figure 2. Flowchart of the sequencing method used in our laboratory. 


\subsection{Array comparative genomic hybridization (aCGH)}

A Roche NimbleGen 12x135K Custom CGH Array was used for dosage analysis. This bespoke $\mathrm{CGH}$ array has been designed to screen for dosage changes within the genes responsible for LQT1-12 (KCNQ1, KCNH2, SCN5A, ANK2, KCNE1, KCNE2, KCNJ2, CACNA1C, CAV3, SCN4B, AKAP9, and SNTA1), the LQT-associated genes GPD1L, KCNE3, SCN1B, $S C N 3 B, C A C N B 2$, and the CPVT1 (RYR2) and CPVT2 (CASQ2) genes.

Two hundred and fifty nanograms of gDNA are processed according to the manufacturer's instructions (NimbleGen Array User's Guide: CGH and CNV Arrays v8.0; http:// www.nimblegen.com). In brief, extracted gDNA from samples and Promega controls are denatured in the presence of a Cy3- (test) or Cy5- (control) labelled random primers and incubated with the Klenow fragment of DNA polymerase, together with dNTPs (5mM of each $\mathrm{dNTP}$ ), at $37^{\circ} \mathrm{C}$ for 2 hours. The reaction is terminated by the addition of $21.5 \mu \mathrm{L}$ of $0.5 \mathrm{M}$ EDTA, prior to isopropanol precipitation and ethanol washing. Following quantification, the test and sex-matched control samples are combined in equimolar amounts and applied to one of the twelve arrays on a microarray slide. Hybridisation is carried out in a Roche NimbleGen Hybridisation Chamber for a period of 48 hours. Slides are washed and scanned using a NimbleGen MS 200 Microarray Scanner. Array image files (.tif) produced by the MS 200 Data Collection Software are imported into DEVA v1.2.1 (Roche NimbleGen Inc) for analysis. Each genomic region exhibiting a copy number change within one of the LQT genes of interest were examined using the UCSC genome browser (http://genome.ucsc.edu/) to determine the location and significance of the change.

\section{Results}

In order to overcome the historical difficulties faced when performing Long QT syndrome testing using postmortem specimens and meet the demand by coroners for rapid results, we addressed the following parameters:

\subsection{DNA purity}

Since poor quality gDNA leads to suboptimal PCR amplification affecting downstream applications, the purity of gDNA is an important criterion for success in generating high quality sequencing data $[14,15]$. A NanoDrop ND-1000 spectrophotometer was used to measure the quality and quantity of the extracted gDNA. The ratio of absorbance at $260 \mathrm{~nm}$ and $280 \mathrm{~nm}$ (A260/280 ratio) is used to assess the purity of gDNA, which should be in the range of 1.8 to 2.0 to be accepted as pure gDNA. The ratio of absorbance at $260 \mathrm{~nm}$ and $230 \mathrm{~nm}$ (A260/230 ratio) is used as a secondary measure of nucleic acid purity, and for pure gDNA should be in the range of 2.0-2.2 [16]. Postmortem gDNA isolated using a standard phenol/ chloroform protease protocol may contain residual phenol, chloroform or ethanol. These contaminants inhibit the activity of DNA polymerase in downstream applications (Figures 3-5) [14], so the purity of the gDNA must be strictly monitored. If a suboptimal A260/280 or A260/230 ratio indicates that the gDNA is of low quality, a secondary cleanup process 
should be considered. In our laboratory, we perform this cleanup using another phenol extraction and ethanol precipitation to further purify the gDNA sample [17].

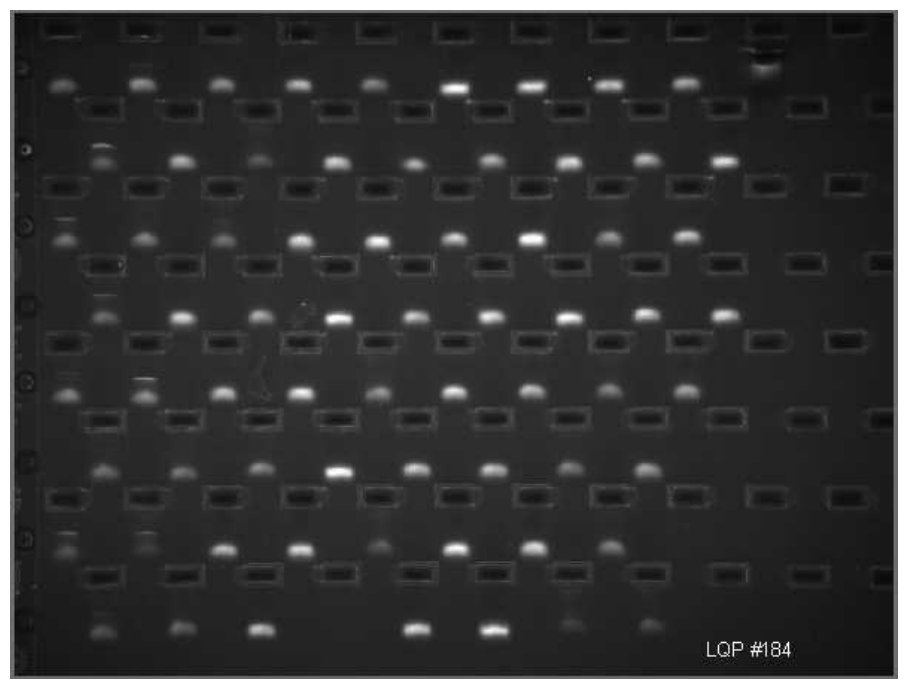

Figure 3. Agarose gel image of PCR amplification from an impure DNA with an acceptable $A_{260 / 280}$ ratio (1.88) but an acceptable $A_{260 / 230}$ ratio (2.5). NOTE: very few exons amplified effectively and strong primer dimers are visible.

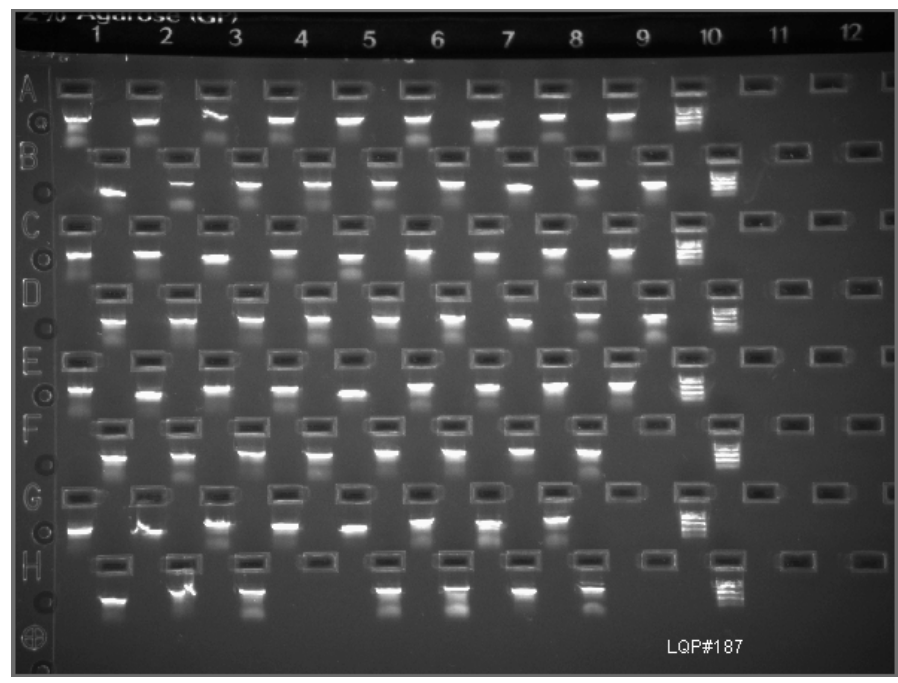

Figure 4. Agarose gel image of PCR products amplified from the same DNA sample after purification. NOTE: successful amplification and significantly reduced primer dimers. 


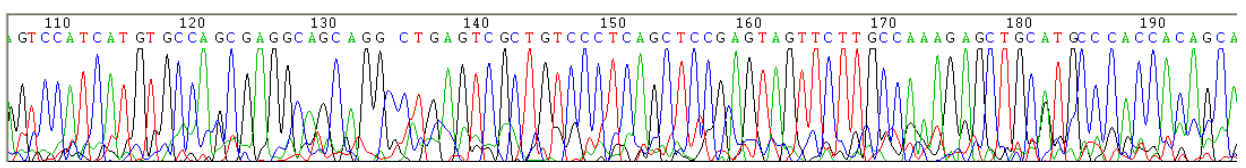

Figure 5. Electropherogram generated from an impure DNA sample with an acceptable $A_{260 / 280}$ ratio (1.95) but an unacceptable $A_{260 / 230}$ ratio (0.95). NOTE: significant baseline noise and data unable to be analysed.

\subsection{Amount of DNA template used in a sequencing reaction}

The extent of dilution of the cleaned PCR product prior to sequencing determines the amount of DNA template used in the sequencing reaction, which can affect the data quality for analysis [15]. The presence of excessive DNA template in a sequencing reaction will lead to rapidly progressive signal loss on the electropherogram (Figure 6), while using insufficient DNA template in a sequencing reaction will result in weak signal strength and a loss of peak shape (Figure 7), causing difficulties in basecalling and accurate analysis [15]. In order to obtain high quality data with good signal strength, the amplified product should be diluted in $\mathrm{ddH}_{2} \mathrm{O}$ to as close as possible to $2.5 \mathrm{ng}$ per $100 \mathrm{bp}$.

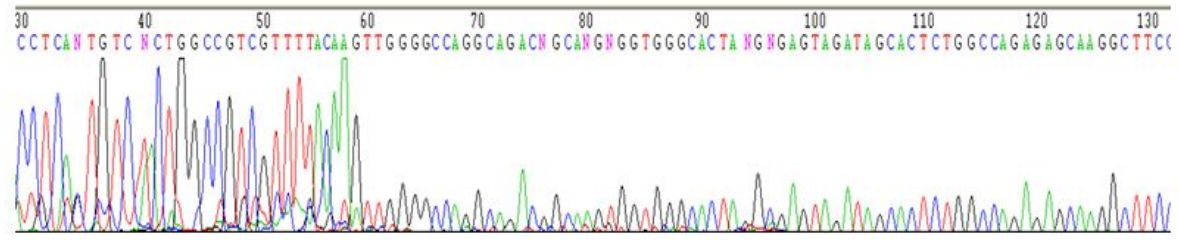

Figure 6. Electropherogram begins with strong high peaks, which fade rapidly.

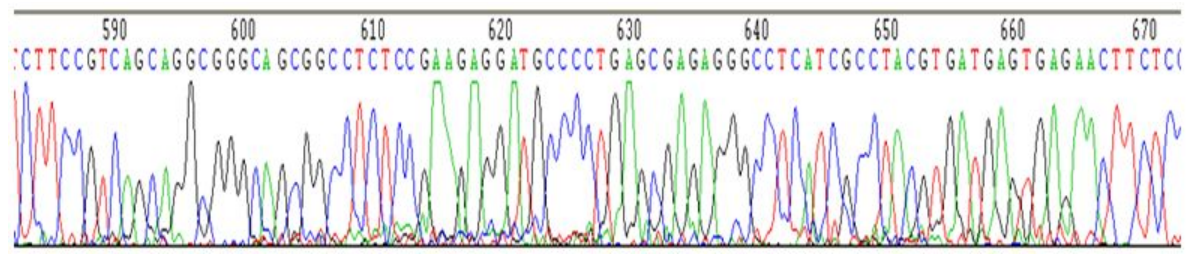

Figure 7. Electropherogram with increased background noise and loss of sharp peak shape. 


\subsection{CleanSEQ treatment of sequencing reactions}

The sequenced product is purified using an Agencourt CleanSEQ system, which uses SPRI (Solid Phase Reversible Immobilization) magnetic bead-based technology. According to the manufacturer's recommendation, the sequencing product should be cleaned using undiluted CleanSEQ reagent, but in our experience, postmortem DNA samples should be cleaned using CleanSEQ reagent diluted 1:2 in $\mathrm{ddH}_{2} \mathrm{O}$ (figures 8,9).

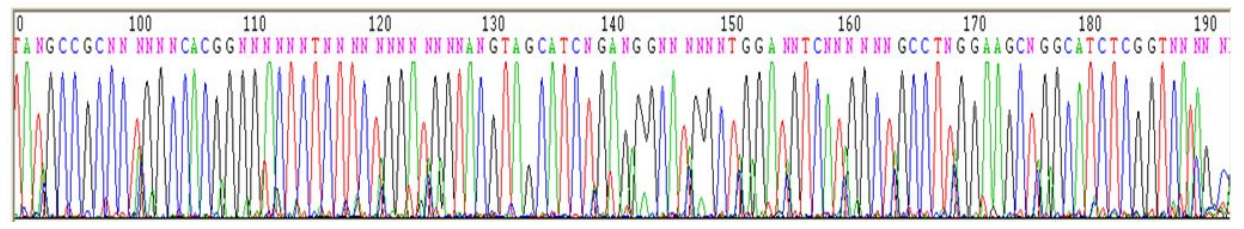

Figure 8. Electropherogram obtained from sequencing product cleaned with $10 \mu \mathrm{l}$ of undiluted CleanSEQ reagent: serious baseline noise and incorrect basecalling.

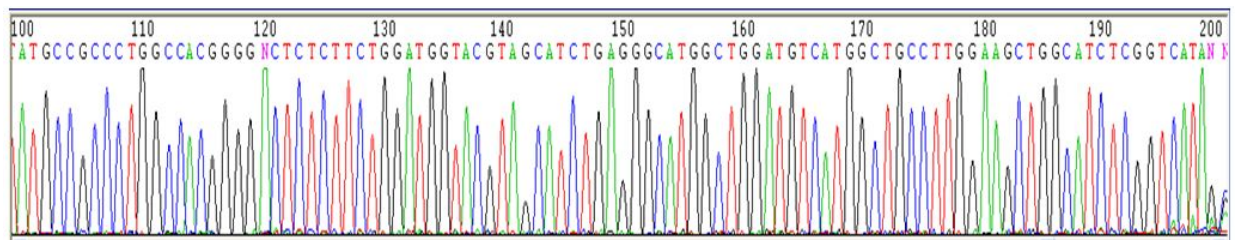

Figure 9. Electropherogram obtained from sequencing product cleaned using diluted CleanSEQ reagent: good quality trace.

\subsection{Drying of DNA-bound beads prior to elution}

The length of drying time following ethanol washing and prior to elution of the sequencing product from the CleanSEQ beads is one of the critical parameters for obtaining high quality sequence data. We assessed 4 different drying periods: 20 minutes, 24 hours, four days and seven days.

A drying time of 20 minutes, as recommended by the manufacturer, is frequently associated with the presence of large 'dye blobs', most probably as a result of residual ethanol in the eluted product (Figure 10); this problem resolves if drying time is extended to 24 hours (Figure 11). However, variable baseline noise on the electropherogram will be evident if the drying time exceeds four days (Figure 12); further increased background noise and loss of resolution occur when the drying time exceeds seven days (Figure 13). 


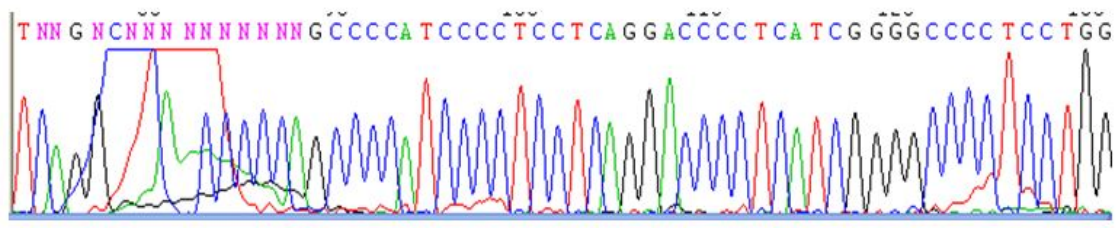

Figure 10. Electropherogram of purified products dried for 20 minutes prior to elution; presence of large dye blob.

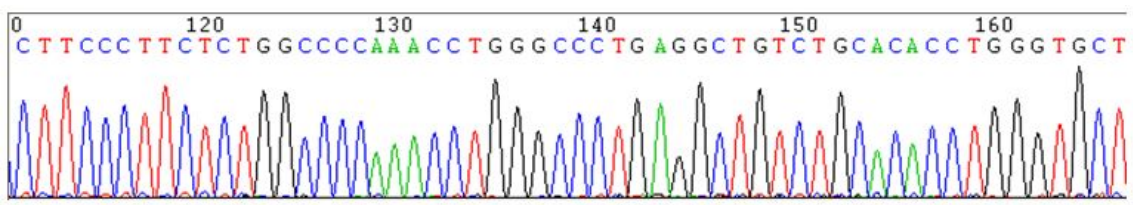

Figure 11. Electropherogram of purified products dried for 24 hours prior to elution; free of dye blobs, minimal baseline noise.

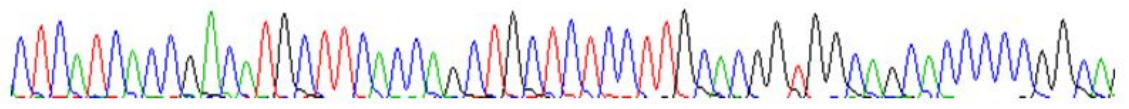

Figure 12. Electropherogram of purified products dried for 4 days prior to elution; presence of shoulders and messy baseline.

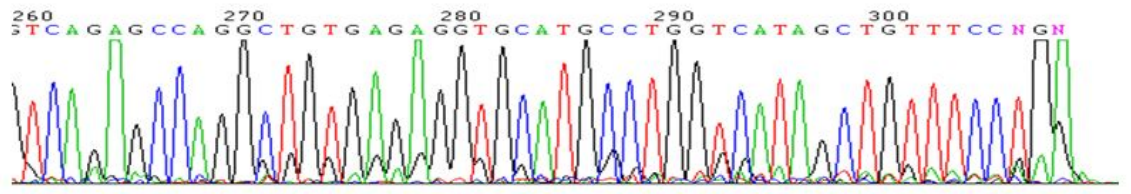

Figure 13. Electropherogram of purified products dried for 7 days prior to elution; extremely messy baseline and early loss of resolution. 


\section{Discussion}

\subsection{DNA quality}

\subsubsection{Sequence analysis}

The gDNA used in a PCR should be as pure as possible in order to optimise the quality of the template that will be used in downstream applications. The purity of DNA is assessed in our laboratory using a NanoDrop ND-1000 spectrophotometer. It is important to consider both A260/280 and A260/230 as poor DNA quality will affect downstream applications $[16,18]$. An unacceptable A260/280 or A260/230 ratio indicates the presence of contaminants in the DNA; an abnormal A260/230 indicates the presence of residual phenol or other chemical from the extraction process, while an abnormal A260/280 most frequently indicates the presence of protein $[16,18]$. If any of the ratios appear to be abnormal, DNA purification should be considered before processing any further.

\subsubsection{Dosage analysis (aCGH)}

The quality requirements for gDNA used in an aCGH assay are not as stringent as those for a sequencing assay. An awareness of the presence of significant degradation is important; however, partial compensation for this can be made by increasing the volume of gDNA used in the amplification and labelling step. In our laboratory, we have found that doubling the volume of gDNA when degradation is visible on the 'check gel' (present in lanes 5-8 of Figure 14) is an effective counter-measure. The adequacy of amplification is measured using a NanoDrop ND-1000 spectrophotometer in order to allow the hybridisation of equimolar amounts of test and control DNA to the array slide. A post-amplification concentration of greater than $2500 \mathrm{ng} / \mu \mathrm{l}$ is ideal for further processing; a sample with a post-amplification concentration of less than $1500 \mathrm{ng} / \mu \mathrm{l}$ is unlikely to produce good quality data for analysis. Therefore, if there is sufficient volume of gDNA available, a repeat amplification with an increased volume of template gDNA should be undertaken.

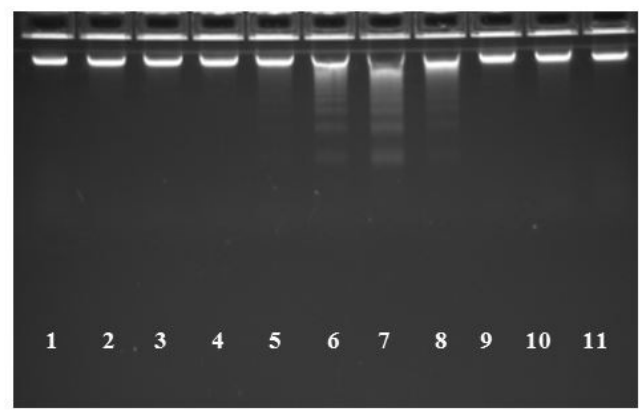

Figure 14. Agarose gel (2\%) check of gDNA quality prior to aCGH. 


\subsection{Amount of DNA template used in a sequencing reaction}

The DNA template should be diluted to $2.5 \mathrm{ng}$ per $100 \mathrm{bp}$ prior to sequencing. Incorrect quantification and dilution will alter the amount of input DNA template in a sequencing reaction, which can lead to problematic data for analysis. In our laboratory, we use Variant Reporter ${ }^{\mathrm{TM}}$ (Applied Biosystems Ltd) for automated analysis of sequence data. The signal strength of the sequence data is automatically assessed. Data with good signal strength is above the value of 200 in the Variant Reporter ${ }^{\mathrm{TM}}$ software. Sequence data with weak signal strength, a value below 100, indicates insufficient DNA template has been used in the sequencing reaction. Noisy background and a loss of sharp peak shape are also commonly evident. A repeat treatment with EXOSAP-IT and less extensive dilution of product is necessary to avoid inaccurate analysis using sequence data with unacceptable quality. In contrast, when excess DNA template is used in a sequencing reaction, a rapidly progressive signal loss is seen on the electropherogram. In this instance, trace quality can be improved by diluting the eluted product with water and reloading the sample on the capillary sequencing platform (we use an Applied Biosystems model 3130xl Genetic Analyzer).

\subsection{CleanSEQ treatment of sequencing reactions}

Agencourt CleanSEQ is routinely used to purify the sequenced products. According to the manufacturer's recommendation, $20 \mu \mathrm{l}$ of sequencing product should be cleaned with $10 \mu \mathrm{l}$ of CleanSEQ reagent. However, we found that this leads to an excess of CleanSEQ beads in relation to the amount of sequence product, therefore unwanted short fragments (e.g unincorporated dNTPs and excess primers) were able to bind to beads during purification, and the quality of sequence data generated was consequently reduced. We found that diluting the CleanSEQ beads 1:2 with $\mathrm{ddH}_{2} \mathrm{O}$ leads to much higher quality data.

\subsection{Drying of DNA-bound beads prior to elution}

This drying step is a critical QC consideration that significantly affects sequence quality. When the purified product is dried for 24 hours prior to elution, the subsequent electropherograms are free of dye blobs, C-shoulders and baseline noise, and consistently pass all QC metrics in Variant Reporter.

A drying time of 20 minutes, as suggested by manufacturer, was found to be problematic, causing the presence of dye blobs. Dye blobs are a common artefact due to either the presence of excess unincorporated dye or residual ethanol following product purification [15]. Previously, operators have focused on purifying sequenced products using an accu- 
rate final concentration of ethanol in an attempt to achieve consistency of evaporation/ drying between sequencing runs. Ethanol is highly volatile and it is therefore difficult to achieve consistency - even the short period of time in which the vessel containing the ethanol is open to allow access can result in a decrease in concentration. Here, we showed that the issue of dye blobs can be resolved by simply extending the drying time to 24 hours, allowing adequate time for all ethanol to evaporate completely, despite any minor differences in the concentration of ethanol used.

On the other hand, we found that the quality of sequence data is adversely affected if the drying time is extended to more than 4 days. This suggests that the stability of purified sequencing products is another factor that affects data quality. High quality sequence data was obtained with a drying time of 24 hours; however, the sequences were suboptimal when elution and electrophoresis were carried out at day 4 or day 7 of drying. This indicates that purified sequencing product is most stable for the first 24 hours and that consideration of the sample stability should be taken into account when aiming for high quality data.

\subsection{Analysis parameters}

\subsubsection{Sequencing}

All sequence traces are analysed in our laboratory using Variant Reporter Software v1.0 (Applied Biosystems). The current CMGS best practice guidelines for Sanger sequence analysis in diagnostic laboratories [19] recommend a PHRED score of at least 20 for bidirectional data (corresponding to $99 \%$ confidence that the base is called correctly), and a PHRED score of at least 30 for unidirectional data $(99.9 \%$ confidence that each base is called correctly). The procedure we describe above produces bidirectional sequence data that meet our laboratory's even more stringent analysis criteria: a Variant Reporter trace score of 35 , corresponding to a false base call rate of $0.031 \%$.

\subsubsection{Dosage analysis}

In order to provide simultaneous dosage analysis of all the Long QT syndrome genes, we developed a custom designed Roche NimbleGen 12x135K CGH array. As part of the validation of this array we analysed twenty patients with known copy number abnormalities [20]. Analysis of the data from these individuals with known copy number changes allowed precise threshold criteria to be developed. It was determined that a $\log _{2}$ ratio $\leq-0.4$ over 6 contiguous probes is indicative of a deletion, and a $\log _{2}$ ratio of $\geq 0.4$ over 15 contiguous probes indicative of a duplication. 


\subsection{Other parameters}

It is important to monitor other QC parameters when it comes to sequence analysis of Long QT specimens: the freshness of the Polymer (POP-7) and the usage of the capillaries in the Applied Biosystems model 3130xl Genetic Analyzer.

Polymer (POP-7) is used to separate DNA fragments on genetic analyzers and the polymer remains stable for up to 7 days [15]. The capillary in the genetic analyzer should be replaced after 1000 injections. Within our laboratory, we perform a regular weekly maintenance of the analyzer, and the capillary is replaced when 600 injections are reached. From past experience, both the freshness of polymer and the usage of the capillary play a vital role in the data quality. Electropherograms with reduced resolution and peak shape are produced when either of these two parameters is suboptimal. In order to achieve high quality data, both freshness of polymer and usage of the capillary should be closely monitored.

\section{Conclusions}

We have successfully established a robust method for processing postmortem specimens for Long QT diagnostic testing in a timely manner. The electropherograms in Figure 15 are indicative of the high quality data routinely produced, despite the limitations inherent in the types of specimens that are referred. The blood sample used to extract gDNA for sequencing in this example was heavily haemolysed on arrival at the laboratory.

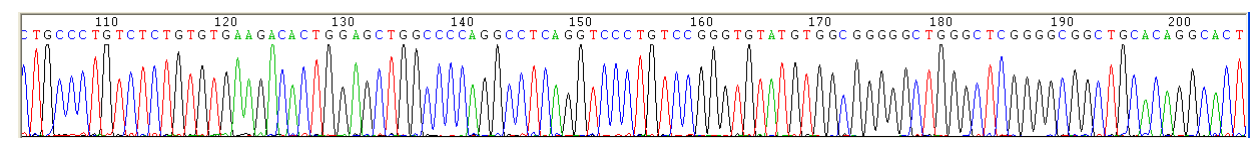

Figure 15. High quality sequence data generated from a haemolysed blood specimen; flat baseline along with distinct and evenly spaced peaks allow accurate basecalling.

The QC parameters described above should be monitored closely in order to consistently achieve optimal results (Figure 16). Although the basic procedure is essentially the same as that routinely used for sequence and aCGH analysis, the poor quality of postmortem specimens as a source of template DNA mean that particular attention needs to be paid to each step, in particular the critical initial assessment of DNA quality, the addition of the appropriate volume of template DNA to the sequencing reaction, and the drying time of the beads used in the purification procedure prior to elution. 


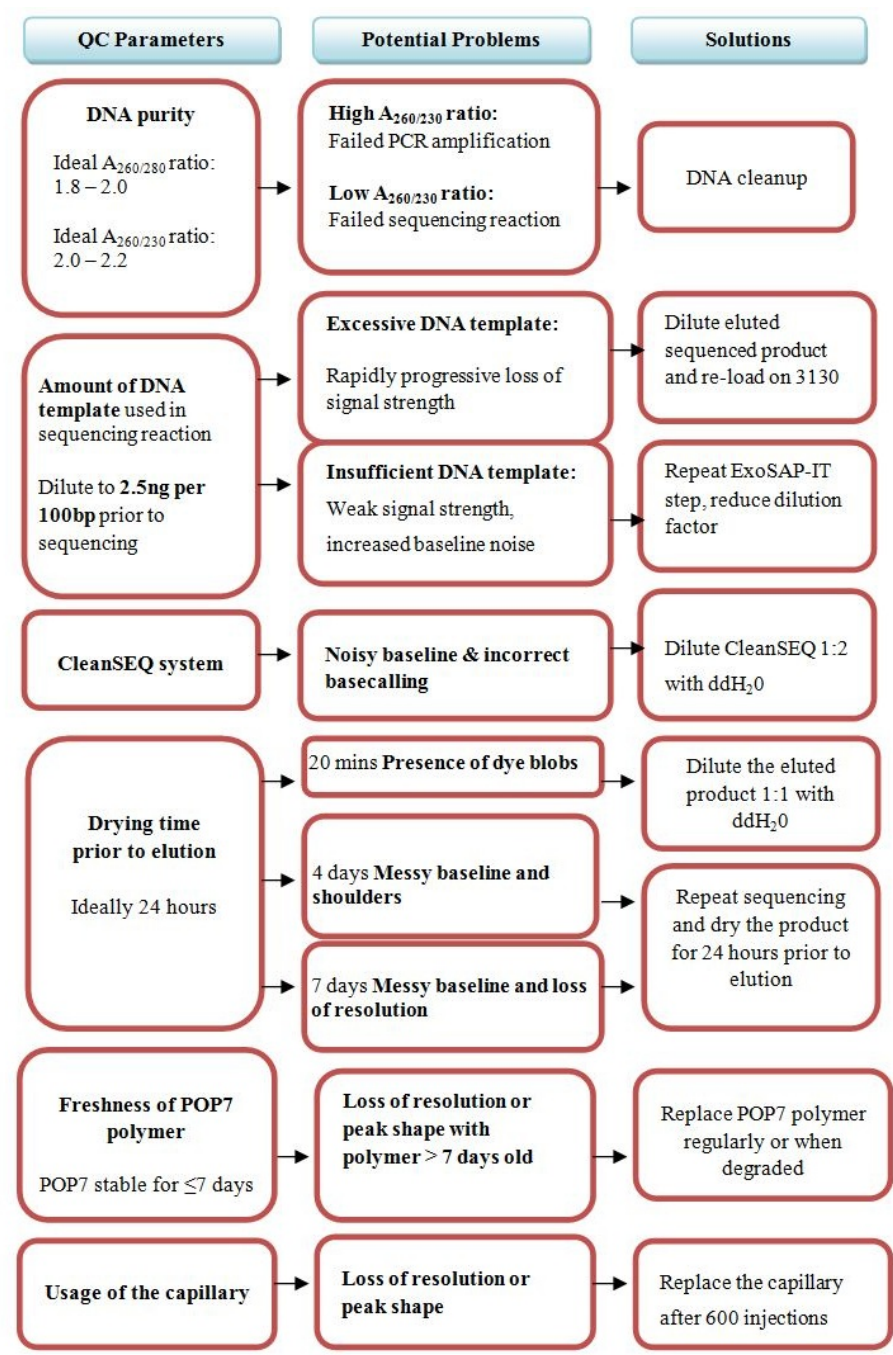

Figure 16. Flowchart of potential QC problems and their solutions.

\section{Acknowledgements}

We acknowledge the assistance of Dr Andrew Dodd in the initial design of primers against the coding regions of the LQT genes, and Mr Daniel Lai for his assistance in optimizing aspects of our amplicon clean-up. 


\section{Author details}

Stella Lai ${ }^{1}$, Renate Marquis-Nicholson ${ }^{1,2^{*}}$, Chuan-Ching Lan ${ }^{1}$, Jennifer M. Love ${ }^{1}$, Elaine Doherty ${ }^{1}$, Jonathan R. Skinner ${ }^{3}$ and Donald R. Love ${ }^{1,2^{*}}$

*Address all correspondence to: donaldl@adhb.govt.nz

1 Diagnostic Genetics, Auckland City Hospital, New Zealand

2 School of Biological Sciences, The University of Auckland, New Zealand

3 Inherited Disease Group New Zealand, Paediatric Cardiac Services, Starship Childrens' Hospital, New Zealand

\section{References}

[1] Hunter, J. D., Sharma, P., \& Rathi, S. (2008). Long QT syndrome. Contin Educ Anaesth Crit Care Pain., 8, 67-70.

[2] Modell, S. M., \& Lehmann, M. H. (2006). The long QT syndrome family of cardiac ion channelopathies: a HuGE review. Genet Med., 8(3), 143-55.

[3] Alders, M., \& Mannens, M. M. A. M. (2003). Romano-Ward Syndrome. Feb 20 [Updated 2012 May 31]., In: Pagon RA, Bird TD, Dolan CR, et al., editors. GeneReviews ${ }^{\mathrm{TM}}$ [Internet]. Seattle (WA): University of Washington, Seattle;, 1993Available from:, http:// www.ncbi.nlm.nih.gov/books/NBK1129/.

[4] Yang, Y., Yang, Y., Liang, B., Liu, J., Li, J., Grunnet, M., Olesen, S. P., Rasmussen, H. B., Ellinor, P. T., Gao, L., Lin, X., Li, L., Wang, L., Junjie, J. X., Liu, Y. Y., Liu, Y. Y., Zhang, S. S., Lian, D. D., Peng, L. Y., Jespersen, T., \& Chen, Y. H. (2010). Identification of a Kir3.4 mutation in congenital long QT syndrome. Am. J. Hum. Genet., 86, 872-880.

[5] Dan, M., \& Roden, M. D. (2008). Long-QT Syndrome. N Engl J Med., 358, 169-176.

[6] Eddy, C. A., MacCormick, J. M., Chung, S. K., Crawford, J. R., Love, D. R., Rees, M. I., et al. (2008). Identification of large gene deletions and duplications in KCNQ1 and KCNH2 in patients with long QT syndrome. Heart Rhythm, 5(9), 1275-1281.

[7] Bonin, S., Petrera, F., Niccolini, B., \& Stanta, G. (2003). PCR analysis in archival post mortem tissues. Mol Pathol., 56, 184-186.

[8] Schwartz, P. J., Stramba-Badiale, M., Crotti, L., Pedrazzini, M., Besana, A., Bosi, G., et al. (2009). Prevalence of the congenital long-QT syndrome. Circulation, 120(18), 1761-1767. 
[9] Skinner, J. R., Crawford, J., Smith, W., Aitken, A., Heaven, D., \& Evans, CA. (2011). Prospective, population-based long QT molecular autopsy study of postmortem negative sudden death in 1 to 40 year olds. Heart Rhythm, 8(3), 412-419.

[10] Gladding, P. A., Evans, C. A., Crawford, J., Chung, S. K., Vaughan, A., Webster, D., et al. (2010). Posthumous diagnosis of long QT syndrome from neonatal screening cards. Heart Rhythm, 7(4), 481-486.

[11] Skinner, J. R. (2010). Sudden Unexplained Death in Infancy and Long QT syndrome. Current Pediatric Reviews, 6, 48-55.

[12] Doherty, E., Marquis-Nicholson, R., Love, J. M., Brookes, C., Prosser, D., \& Love, D. R. (2011). Primer Design to Sequence Analysis- a Pipeline for a Molecular Genetic Diagnostic Laboratory, Applications and Experiences of Quality Control, Ognyan Ivanov (Ed.). 978-9-53307-236-4, InTech, Available from, http://www.intechopen.com/ books/applications-and-experiences-of-quality-control/primer-design-to-sequenceanalysis-a-pipeline-for-a-molecular-genetic-diagnostic-laboratory.

[13] Lai, D., \& Love, D. R. (2012). Automation of a primer design and evaluation pipeline for subsequent sequencing of the coding regions of all human Refseq genes. Bioinformation, 8(8), 363-366.

[14] Verhagen, O. J., Wijkhuijs, A. J., van der Sluijs-Gelling, A. J., Szczepanski, T., van der Linden-Schrever, B. E., Pongers-Willemse van, M. J., Wering, E. R., van Dongen, J. J., \& van der Schoot, C. E. (1999). Suitable DNA isolation method for the detection of minimal residual disease by PCR techniques. Leukemia, 13(8), 1298-1299.

[15] Applied Biosystems Chemistry Guide, second edition. (2009). DNA Sequencing by Capillary Electrophoresis. Available from:, http:// www3.appliedbiosystems.com/cms/groups/mcb_support/documents/generaldocuments/cms_041003.pdf.

[16] T009 - Technical Support Bulletin and 260/230Ratios. (2008). Available from:, http:// www.nanodrop.com/Library/T009-NanoDrop\%201000-\&-NanoDrop\%208000-Nucleic-Acid-Purity-Ratios.pdf.

[17] Powell, R., \& Gannon, F. (2002). Purification of DNA by phenol extraction and ethanol precipitation. Oxford Practical Approach Series, Oxford University Press., Available from:, http://fds.oup.com/www.oup.co.uk/pdf/pas/9v1-7 -3.pdf.

[18] T042 - Technical Bulletin. (2011). Assessment of Nucleic Acid Purity. Available from:, http://www.nanodrop.com/Library/T042-NanoDrop-Spectrophotometers-NucleicAcid-Purity-Ratios.pdf.

[19] Ellard, S., Charlton, R., Yau, M., Gokhale, D., Taylor, G., Wallace, A., \& Ramsden, S. C. (2009). Practice guidelines for Sanger Sequencing Analysis and Interpretation. Available from:, http://www.cmgs.org/BPGs/pdfs\%20current\%20bpgs/Sequencingv2.pdf. 
[20] Marquis-Nicholson, R., Doherty, E., Thrush, A., Love, J. M., Lan-C, C., George, A. M., \& Love, D. R. (2012). Array-based identification of copy number changes: simultaneous gene-focused and low resolution whole genome analysis. Sultan Qaboos University Medical Journal, submitted. 